\title{
28 Policy framework for the use of therapy dogs in educational settings
}

\author{
Christine Grove, Linda Henderson, and Felicia Lee
}

\section{How to use this policy}

There is increased interest in the use of therapy dogs in schools. The research is showing increases in wellbeing for those involved in such programs. Currently, there are a small number of schools with therapy dog programs. With the increasing interest in such programs, there is a need for strong policy frameworks to ensure success. This policy example is attending to this need and is based on the preliminary evidence of a survey of schools that either had a therapy dog program or were seeking to have one. Analysis of the survey has informed the development of this policy framework.

This policy provides guidelines for assessing a school's readiness for a therapy dog program, key factors for consideration for implementation, and principles to manage common challenges associated with using therapy dogs in educational settings. The policy serves as a set of recommendations that were developed based on two studies conducted with schools in Victoria, Australia and informed by existing research on the use of therapy dogs in educational settings in the United States, Canada, and the United Kingdom. As such, this policy example is intentionally broad and school policy creators are encouraged to customise strategies outlined in this policy to fit within their unique school context.

The implementation of a therapy dog program in a school involves several considerations ranging from consent and liability, hygiene, safety, and cultural and personal factors to considerations for student learning and wellbeing outcomes. As such, leadership and whole-school support and commitment are essential in its successful implementation (Lane \& Zavada, 2013). A school team including school leaders, key staff overseeing students' learning and wellbeing, as well as potential therapy dog handler(s), should be involved in developing this policy. This policy example is intentionally broad and school policy creators are encouraged to delete and build upon suggestions below to create a policy that best represents their school.

[To adapt and use this policy, delete or modify the text as indicated] 


\section{[INSERT name of school] Therapy dog implementation policy}

\section{Rationale}

Therapy dogs are increasingly used in educational contexts such as in primary schools, secondary schools, and universities across Australia. There is preliminary evidence indicating that therapy dogs can promote wellbeing, reduce physiological signs of stress, provide social support, encourage pro-social behaviour, motivate participation and engagement in activities, act as a social catalyst to facilitate positive social interactions in children and complement professional therapies (Jalongo \& Petro, 2018). Despite the potential benefits of therapy dogs, there are several concerns and challenges that might arise from successfully introducing a therapy dog into a school context. Primary concerns by schools include legal implications and liability, allergy concerns, hygiene and sanitation concerns, safety concerns (e.g., dog's aggression towards students), cultural differences, fear of dogs, animal welfare, and funding associated with animal maintenance and program implementation (Anderson, 2007; Friesen, 2010; Henderson et al., 2020; Jalongo, 2005; Jalongo, Astorino, \& Bomboy, 2004; Jalongo \& Petro, 2018; Lane \& Zavada, 2013; Sheckler, 2017)

Presently there are limited policies or guidelines on implementing a therapy dog program within educational settings, and they are often not based on evidence. While there are a number of guidelines on animal-assisted interventions in other settings such as healthcare facilities (American Veterinary Medical Association, 2014; Freeman et al., 2016; Lefebvre et al., 2008; Lenihan et al., 2016; Linder et al., 2017; Murthy et al., 2015), these tend to focus heavily on the reduction of allergies and measures to improve hygiene as well as sanitation. Some therapy dog organisations have guidelines on the administrative structure, standards of practice, training of dog and handler teams, documentation, and sample forms on the use of therapy dogs. While these are important and applicable to some extent in the school context, they do not cover the full range of issues stated above that are faced by schools implementing therapy dog programs. In addition, policy developed by National Departments of Education across Australia's states and territories focus on the use of animals for teaching (e.g., during science lessons) with brief information emphasising the importance of animal welfare, safety, hygiene, and sanitation procedures when interacting with animals in other contexts (e.g., school excursions, classroom pets). As such, a customised policy unique to the school context should be developed to navigate the processes involved in therapy dog program implementation.

\section{What is a therapy dog?}

A therapy dog refers to a dog trained to provide therapeutic benefit mainly through Animal-Assisted Therapy (AAT), Animal-Assisted Intervention (AAI), or Animal-Assisted Education (AAE) (Jalongo \& Petro, 2018). Therapy dogs are first selected for their temperament and subsequently trained together with 
their handler, usually by therapy dog organizations, to be calm and obedient such that they are able to provide emotional support, comfort and companionship to different individuals in various educational, health care, and community settings (Jalongo \& Petro, 2018). It is also worth noting that therapy dogs are distinctively different from service or guide dogs, with the latter trained for the purpose of meeting the specific needs of one individual in the long term. Overall, there is much variability in how a "therapy dog" is involved, and this is largely dependent on their handler (e.g., their professional practice/expertise), the setting they are involved in (e.g., education, hospitals, courtrooms), and the goals (e.g., support learning and education, promote mental health, encourage positive social interactions, facilitate specific therapeutic outcomes) (Jalongo \& Petro, 2018).

\section{Purpose}

This policy framework aims to help schools assess their own readiness for a therapy dog program and be cognisant of key factors for consideration for implementations, as well as to provide schools with recommendations to manage common challenges and concerns associated with using therapy dogs in educational settings. The framework can be incorporated within an existing school's overall wellbeing policy where therapy dogs are not a program that occurs on the side but rather as part of a whole school approach. The policy framework can be adapted to suit the specific needs of the context it will be used.

\section{Scope}

The policy framework for the use of therapy dogs applies to current students, teachers, staff, school leaders, and parents of [INSERT name of school].

[INSERT specific roles and responsibilities of individuals or teams]

\section{Policy statement}

[INSERT name of school] policy outlines the following:

- Factors of consideration of key stakeholders involved in a therapy dog program - therapy dog handlers, school leaders and staff, students, and parents;

- Common concerns and challenges associated with implementing a therapy dog program.

Key Stakeholders include:

\section{Therapy dog handlers}

[INSERT name of school] therapy dog handlers will ensure that their dogs receive appropriate certified training where they are rigorously trained and 
evaluated to be reliably non-aggressive to both people and other dogs regardless of circumstances, highly adaptable, and able to interact easily with people.

During the therapy dog training, [INSERT name of school] handlers are also trained to meet welfare, safety, and hygiene requirements for both the dog and students, and to use the dog effectively.

[INSERT name of school] handlers will be prepared to:

a be personally and financially responsible for the dog's welfare and maintenance including safety, feeding, grooming, cleaning, and vaccination. In the event that the dog is involved in school programs regularly or in the long term, it is recommended for the handler to request financial support from the school since the dog is part of an intervention employed for meeting the students' needs (Anderson, 2007). In such cases, it is important to put the financial plans into a written budget outlining a list of all expenses required in order to deliver the program and share them with all responsible parties (Jalongo, 2005).

b be vigilant in identifying signs and triggers of injury, distress, or exhaustion for their dogs and be able to respond accordingly. Regular breaks should be given to the dog. Suspension of the program may be required if the dog shows a negative behavioural change, fearful behaviour during interactions, or has medical concerns.

c (c)trouble-shoot if an incident occurs (e.g., when a student has a negative response to a dog), and adopt appropriate measures when needed (e.g., removal of the dog, medical care, debrief with student).

Efforts are made at [INSERT name of school] to facilitate successful implementation of therapy dog programs in schools. Handlers should be proactive in researching on the current evidence base of the use of therapy dogs and communicating with others who have had experience implementing such a program (Jalongo, 2005).

Handlers at [INSERT name of school] will have a good understanding of how therapy dogs are incorporated/included in their educational context as well as their impact.

[INSERT name of school] establishes a clear goal/purpose of the inclusion of a therapy dog in different school activities - e.g., desired outcomes, who might benefit, and how (Anderson, 2007; Freeman et al., 2016; Lane \& Zavada, 2013).

[INSERT name of school] facilitates planning of therapy dog activities with key school personnel (e.g., frequency and duration of activities, how the dog may be incorporated safely and appropriately, anticipated risks and concerns) as well as the evaluation of outcomes.

\section{School leaders and staff}

Leadership and whole-school support at [INSERT name of school] are essential in the successful implementation of a therapy dog program (Lane \& Zavada, 2013). 
Efforts are made at [INSERT name of school] to support school staff's acceptance of the therapy dog. Inviting the therapy dog into a school should not be a unilateral decision.

[INSERT name of school], endeavours to consider and discuss the thoughts, concerns, and ideas of all stakeholders, and these should be informally solicited by conducting preliminary meetings with administrators, parents, teachers, paraprofessionals, and students early on to:

a Briefly explain the idea of involving a therapy dog in school and goals;

b Obtain initial support;

c Learn of any dog-related allergies or phobias;

d Discuss and address any other concerns.

[INSERT name of school] therapy dog program develops a therapy dog implementation user guide that identifies and explains how the dog will be included in the classroom and school activities (Anderson, 2007)

Stakeholders [INSERT name of school] are likely to have varied questions. Our [INSERT name of school] endeavours to hold an information session or send out a newsletter introducing the therapy dog program by preparing information ahead of time (e.g., benefits of therapy dogs, their inclusion in schools), providing opportunities to ask questions, and preparing to respond to any potential concerns (Linder et al., 2017).

Our [INSERT name of school] endeavours to obtain support from most of the school community ahead of time and presenting collaboratively to school staff is recommended (Anderson, 2007)

[INSERT name of school] has the presence of 1 or 2 other school staff who are involved in the therapy dog program to ensure that the handler is not the sole person managing the program.

[INSERT name of school] has considerations such as appropriate indoor and outdoor areas for the dog and scheduling of activities and breaks for the dog.

[INSERT name of school] has school-wide protocols to address sanitation and safety of the program and has provided training of school staff and students on appropriate ways to interact with the dog and the training of emergency protocols (e.g., in the event of dog scratch or bite, students' adverse reactions).

[INSERT name of school] has made efforts to establish communication plans for engaging parents and students about the program, to address questions about the therapy dog program (e.g., cultural differences, fear of dogs, allergies, or medical concerns), and to obtain consent and assent.

Efforts are made at [INSERT name of school] to provide adequate planning and preparation for introducing the therapy dog to school staff so that they are educated on appropriate animal care and behavioural expectations and are able to step in when necessary in times of emergencies (e.g., negative dog reactions in students, emergency protocols). 


\section{Students}

[INSERT name of school] regularly assesses the needs and suitability of students who the therapy dog program will be working with. This is critical to ensure that the program goals are met and student welfare is considered. At [INSERT name of school] we will:

- Determine which students are to participate in the therapy dog program (e.g., which students would benefit the most from this program? How should the program be structured to best meet their needs [e.g., whole class, groups, or individually]? How does a therapy dog program add value to existing programs in meeting the needs of these students? How would the program fit into or complement the usual curriculum and schedule?);

- Provide suitable alternatives for children who are unable to participate (e.g., for cultural or religious reasons, allergies, fear of dogs) (Jalongo, 2005);

- Encourage families to participate (Jalongo, 2005);

- Provide adequate planning and preparation for introducing the therapy dog to students so that they are educated on appropriate animal care and behavioural expectations.

\section{Parents/primary caregivers}

At [INSERT name of school] early engagement with parents/primary caregivers is essential once there is clear direction that a therapy dog program may be introduced in school (Anderson, 2007; Friesen, 2010; Jalongo, 2005).

At [INSERT name of school] we endeavour to inform parents/primary caregivers of procedures and processes and to provide them with the opportunity to ask questions and voice concerns. We ask that parents/primary caregivers provide written consent signifying they understand and support the dog's inclusion (Anderson, 2007).

At [INSERT name of school] we will ensure that there is ample time to engage parents before the commencement of a therapy dog program. Parents who provide consent to their child's participation will be provided with additional information about the program and have opportunities to have any concerns addressed. Parents who do not consent will be provided with information on how their child will be engaged in alternative ways (Jalongo, 2005).

[INSERT name of school] has assessed our own readiness for a therapy dog program by considering program indicators (i.e., the characteristics of a therapy dog program) and implementation site indicators (i.e., characteristics of the school, students, and families/communities involved) (National Implementation Research Network, 2018). 


\section{Rating of evidence base}

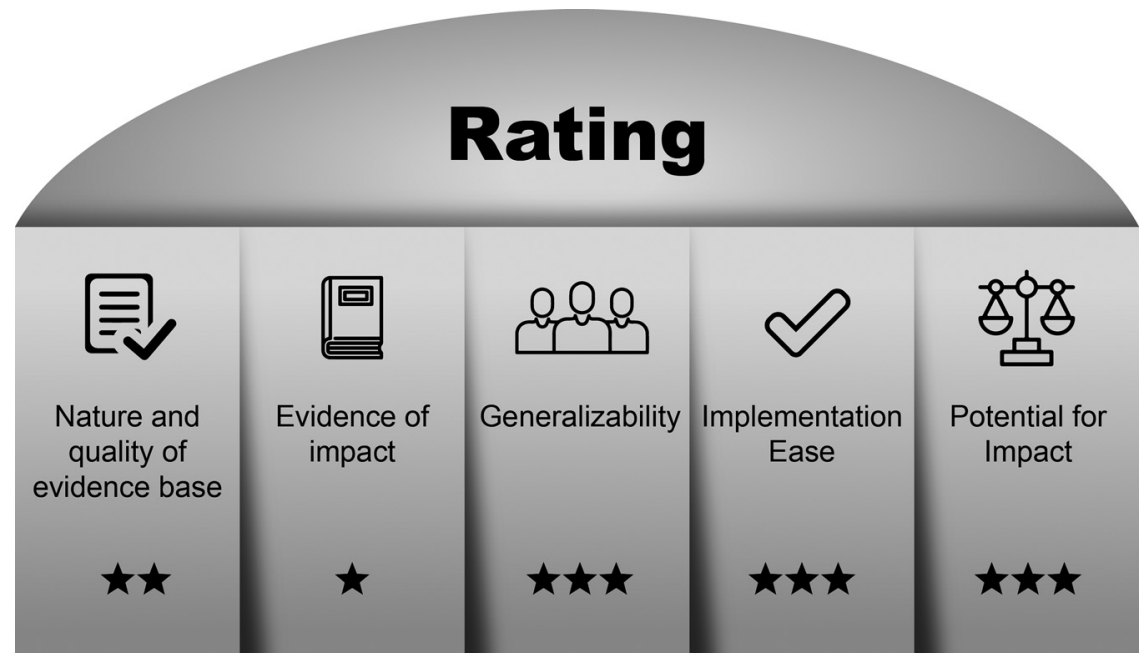

Figure 28.1. The Use of Therapy Dogs in Educational Settings Rating of Evidence.

Author Note. The evidence base on the impact and implementation of therapy dog programs in educational settings is still evolving. The current policy framework was developed based on preliminary findings based on research conducted with schools and therapy dog organisations from Victoria (Australia), as well as informed by researchers in the field who are studying the impact of therapy dogs in educational settings. There has not been any formal evaluation of these recommendations outlined in the policy framework. Recommendations outlined in the policy framework are informed by evidence and research, schools, therapy dog organisations, and researchers who have implemented therapy dog programs in various ways in schools and within different school contexts. Implementation of the policy may require additional resourcing or funding. For example, decisions will need to be made about the ownership of the therapy dog and therefore responsibility for costs involved in this ownership. Other resourcing decisions might include designated space for the dog to rest and relieve itself and hygiene facilities such as hand sanitiser for students post involvement with the dog. From our own research, potential impact that may arise from the implantation of the policy is strong with significant improvements in wellbeing noted across a wide age range of research participants.

\section{Authorship}

Dr Christine Grove, Monash University

Dr Linda Henderson, Monash University

Felicia Lee, Monash University

[INSERT RELEVANT STAFF MEMBERS]

\section{Related policy and documents}

[INSERT RELEVANT POLICY AND DOCUMENTS] 
Wellbeing policies

Emergency, safety, and hygiene policies

Animal welfare policies

Victoria State government school policy: Teaching with animals: https:// www.education.vic.gov.au/school/principals/spag/curriculum/Pages/animals. aspx

\section{Date of ratification}

This policy was ratified on the [INSERT DATE].

\section{Date of review}

This policy will be reviewed by [INSERT DATE].

\section{Further reading}

Henderson, L., Grové, C., Lee, F., Trainer, L., Schena, H., \& Prentice, M. (2020). The impact of the Story Dogs reading program on student wellbeing: Report for Story Dogs (Version 1). Monash University. https://doi.org/10. 26180/5e48f4427390d

Jalongo, M. R. (2018). Children, dogs and education: Caring for, learning alongside, and gaining support from canine companions. Cham: Springer International Publishing.

National Implementation Research Network. (2018). The hexagon: An exploration tool.https://nirn.fpg.unc.edu/sites/nirn.fpg.unc.edu/files/resour ces/HeadStart-HexagonDiscussionandAnalysisTool.pdf

\section{Expert consultation}

Schools may consider consultations with local therapy dog organisations who work closely with educational settings, consultations with schools who have already implemented therapy dog programs, as well as consultations with academics researching the implementation of therapy dog programs in schools for further support.

\section{References}

American Veterinary Medical Association. (2014). Guidelines for animal assisted activity, animal-assisted therapy and resident animal programs. https://ebusiness. avma.org/files/productdownloads/guidelines_AAA.pdf

Anderson, A. K. (2007). Who let the dog in? How to incorporate a dog into a self contained classroom. TEACHING Exceptional Children Plus, 4(1). https://files.eric. ed.gov/fulltext/EJ967469.pdf 


\section{2}

Grove, Henderson, \& Lee

Freeman, L., Linder, D., Mueller, M., \& Gibbs, D. (2016). Animal-assisted interventions: How-to guide for facilities. http://hai.tufts.edu/paws/for-facilities-seeking-therapyanimals/

Friesen, L. (2010). Exploring animal-assisted programs with children in school and therapeutic contexts. Early Childhood Education Journal, 37(4), 261-267.

Jalongo, M. R. (2005). "What are all these dogs doing at school?": Using therapy dogs to promote children's reading practice. Childhood Education, 81(3), 152-158.

Jalongo, M. R., Astorino, T., \& Bomboy, N. (2004). Canine visitors: The influence of therapy dogs on young children's learning and well-being in classrooms and hospitals. Early Childhood Education Journal, 32(1), 9-16.

Jalongo, M. R., \& Petro, J. (2018). Promoting children's well-being: Therapy dogs. In M. R. Jalongo (Ed.), Children, dogs and education: Caring for, learning alongside, and gaining support from canine companions (pp. 179-209). Cham: Springer International Publishing.

Lane, H. B., \& Zavada, S. D. W. (2013). When reading gets ruff: Canine-assisted reading programs. The Reading Teacher, 67(2), 87-95.

Lefebvre, S. L., Golab, G. C., Christensen, E. L., Castrodale, L., Aureden, K., Bialachowski, A., ... Weese, J. S. (2008). Guidelines for animal-assisted interventions in health care facilities. American Journal of Infection Control, 36(2), $78-85$.

Lenihan, D., McCobb, E., Diurba, A., Linder, D., \& Freeman, L. (2016). Measuring the effects of reading assistance dogs on reading ability and attitudes in elementary school children. Journal of Research in Childhood Education, 30(2), 252-259.

Linder, D. E., Siebens, H. C., Mueller, M. K., Gibbs, D. M., \& Freeman, L. M. (2017). Animal-assisted interventions: A national survey of health and safety policies in hospitals, eldercare facilities, and therapy animal organizations. American Journal of Infection Control, 45(8), 883-887.

Murthy, R., Bearman, G., Brown, S., Bryant, K., Chinn, R., Hewlett, A., ... Weber, D. J. (2015). Animals in healthcare facilities: Recommendations to minimize potential risks. Infection Control \& Hospital Epidemiology, 36(5), 495-516.

National Implementation Research Network. (2018). The hexagon: An exploration tool. https://nirn.fpg.unc.edu/sites/nirn.fpg.unc.edu/files/resources/HeadStart-Hexa gonDiscussionandAnalysisTool.pdf

Sheckler, K. A. (2017). The effect of therapy dogs on children in a learning environment. (Unpublished Master's thesis). Rowan University, New Jersey. 